\title{
Chemical Agents
}

National Cancer Institute

\section{Source}

National Cancer Institute. Chemical Agents. NCI Thesaurus. Code C20190.

The consideration of chemical factors as a variable in disease incident, transmission, and control. 